\title{
Empresa e instituições na teoria schumpeteriana: influências do ambiente social sobre a inovação e o progresso tecnológico
}

Ronivaldo Steingraber ${ }^{1}$

Resumo: Este artigo analisa a influência da relação entre instituições e capital social no processo de inovação da empresa na teoria Schumpeteriana. A empresa inovadora necessita desenvolver competências e habilidades para lidar com a inovação, absorvendo conhecimento e interagindo com o ambiente. Tal ambiente ultrapassa a fronteira do mercado e se transforma em um fator social, denominado por Nelson e Sampat (2001) de tecnologia social. Este artigo busca contribuir com a aproximação da teoria econômica Schumpeteriana da inovação com a Sociologia Econômica.

Palavras-chave: Instituições. Capital Social. Inovação. Progresso Tecnológico. Tecnologia Social.

JEL: A13, O30, O33

\section{Firm and institutions in Schumpeterian theory: influences of social environment on innovation and technological progress}

Abstract: This article examines the influence of external environment in the technological progress and developing innovations in the Schumpeterian theory. The innovative firm needs to develop competencies and skills to deal with innovation, absorbing knowledge and interacting with the environment. Such this environment beyond the boundary of the market and becomes a social factor, called by Nelson e Sampat (2001) of social technology. This article seeks to contribute to the rapprochement of Schumpeterian theory of innovation with Economic Sociology. Keywords: Institutions, Social capital, Innovation, Technological Progress, Social Technology.

JEL: A13, O30, O33

1 Prof. Universidade Federal de Santa Catarina - UFSC. Doutor em desenvolvimento econômico pela UFPR. e-mail: ronivaldo.ufsc@gmail.com 


\section{Introdução}

A estratégia de inovação mostra-se presente em diversas áreas de negócio, principalmente com o advento da globalização e da flexibilização produtiva. Por outro lado, a teoria econômica tradicionalmente pouco considera estes efeitos. Este artigo explora os principais fatores de inovação sistêmica na teoria Schumpeteriana e de sua absorção do conceito de ambiente social, como o conceito de instituições e de capital social no estudo do processo de inovação.

O estudo do impacto econômico das inovações e do progresso tecnológico na produção industrial não se limita a fatores econômicos relacionados às causas das inovações e a evolução da tecnologia. A teoria Schumpeteriana e a evolucionária ${ }^{1}$ incorporaram definições importantes que destacam o papel do ambiente social no qual a empresa, ou o empresário inovador/empreendedor, está inserida.

O desenvolvimento desta teoria econômica incorporou elementos novos na caracterização do ambiente externo à empresa. Inicialmente, denominou-se de instituição toda a estrutura física, valores, costumes e demais fatores sociais inerentes ao progresso científico, tecnológico e ao desenvolvimento de inovações. Atualmente, a teoria preocupa-se também com o desenvolvimento do conceito de capital social na determinação da capacidade de inovação da economia, visto que o papel das instituições, e do capital social, já é considerado decisivo na capacidade de interação das empresas com o conhecimento, ao gerar as capacidades, internas e externas, de promoção das inovações.

Este artigo busca identificar as competências da empresa inovadora com o ambiente econômico e social, com destaque para o papel das instituições e do capital social. Para tanto, quatro seções são apresentadas, contabilizando esta introdução. A segunda seção analisa a relação entre as empresas e as instituições na teoria Schumpeteriana. O capítulo três analisa a relação entre a empresa inovadora e a formação de capital social com os atores envolvidos no processo de inovação, sejam eles econômicos, sejam sociais. A seção quatro conclui o artigo.

\section{Inovações e instituições}

O estudo do impacto das instituições sobre a atividade econômica é antigo e envolve a capacidade de geração de inovações no mercado. A teoria Schumpeteriana incorporou o tema na sua agenda de pesquisa e discute com outras escolas, como a Economia Institucional e a Nova Economia Institucional e agora a Sociologia Econômica, o papel das instituições no processo de inovação. $\mathrm{O}$ foco nas instituições revela uma preocupação com a explicação de como o conhecimento é formado na sociedade e ultrapassa os limites teóricos

1 Definimos as duas teorias apenas como teoria Schumpeteriana a partir daqui. 
STEINGRABER, R. Empresa e Instituições na Teoria Schumpeteriana: Influências do Ambiente....

e práticos do mercado imputado na teoria econômica ortodoxa².

Nos últimos anos incorporou-se o uso do conceito de capital social para definir o ambiente social no qual o conhecimento é gerado. O capital social estabelece as redes de contatos entre os indivíduos, disseminando conhecimento tecnológico. Estes dois conceitos serão trabalhados a seguir, dentro das leituras da própria escola de pensamento econômico. O quadro 1 a seguir resume o impacto dos dois ambiente sobre a empresa inovadora.

QUADRO 1: IMPACTO DAS CARACTERÍSTICAS SETORIAIS E INSTITUCIONAIS SOBRE O PROCESSO DE INOVAÇÃO

\begin{tabular}{|c|c|}
\hline Característica & Impacto sobre a inovação \\
\hline \multicolumn{2}{|l|}{ Instituições formais: } \\
\hline $\begin{array}{l}\text { Estrutura legal: leis, direito de } \\
\text { propriedade, contratos, etc; }\end{array}$ & $\begin{array}{l}\text { Garantem a propriedade do conhecimento e a } \\
\text { apropriação do lucro da inovação; }\end{array}$ \\
\hline $\begin{array}{l}\text { Atores: governo, sistema finan- } \\
\text { ceiro, universidades, etc; }\end{array}$ & $\begin{array}{l}\text { Garantem o funcionamento das regras (es- } \\
\text { trutura legal), geração e disponibilização de } \\
\text { recursos para a P\&D\&I (conhecimento, finan- } \\
\text { ciamento, etc); }\end{array}$ \\
\hline $\begin{array}{l}\text { Instituições informais: costu- } \\
\text { mes, hábitos, etc; }\end{array}$ & $\begin{array}{l}\text { Impactam o mercado de trabalho (capital hu- } \\
\text { mano), a formação de contratos (capital so- } \\
\text { cial) e o hábito de consumo (demanda); }\end{array}$ \\
\hline \multicolumn{2}{|l|}{ Capital social formal: } \\
\hline $\begin{array}{l}\text { Extensão: número de atores, } \\
\text { número de pesquisadores em } \\
\text { determinada área de conheci- } \\
\text { mento; }\end{array}$ & $\begin{array}{l}\text { Efeito de escala e aumento do conhecimento } \\
\text { acumulado geram mais inovações. As imita- } \\
\text { ções também podem aumentar; }\end{array}$ \\
\hline $\begin{array}{l}\text { Densidade: volume de conheci- } \\
\text { mento acumulado transmitido } \\
\text { entre os atores; }\end{array}$ & $\begin{array}{l}\text { Aumento do capital social, conduz ao aumen- } \\
\text { to do volume e do impacto das inovações; }\end{array}$ \\
\hline Capital social informal & $\begin{array}{l}\text { Aumento da circulação do conhecimento, au- } \\
\text { mento do número de inovações, mas pode re- } \\
\text { duzir o impacto econômico pelo aumento da } \\
\text { imitação }\end{array}$ \\
\hline
\end{tabular}

FONTE: O autor (2009).

O papel das instituições no processo de desenvolvimento econômico foi considerado há muito tempo pela teoria econômica. A compreensão do processo de formação das mesmas e sua dinâmica ainda são desconhecidas por muitos

2 A Economia Neoclássica assume pressupostos de equilíbrio, eficiência de mercados e uma visão do homus economicus que possui conhecimento completo e desta forma, pouco se contribui com outras ciências, como a Administração ou a Sociologia. Teorias como a Institucionalista, nova e antiga, bem como a Schumpeteriana, apresentam maior relevância na definição da empresa moderna. 
STEINGRABER, R. Empresa e Instituições na Teoria Schumpeteriana: Influências do Ambiente....

economistas ortodoxos ${ }^{3}$ que por vez, assumem os efeitos do ambiente sócio econômico como exógenos, debruçando-se na análise dos efeitos, positivos ou negativos, das instituições sobre o sistema econômico.

Conforme Nelson e Sampat (2001), a definição de instituição é abrangente e envolve diferentes entendimentos sobre a constituição e o papel das mesmas. Para os autores, a definição de instituição permeia o conceito de "tecnologias sociais". Para outros autores, como Williamson, Coase, Veblen, Commons e Hayek elas apresentam definições que vão desde as 'estruturas de governança', 'custos e transação', 'regras do jogo', 'comportamento cooperativo' e 'práticas da sociedade' respectivamente.

A influência das instituições sobre o processo de inovação da economia não foge à regra. A partir do trabalho de Edquist e Johnson (1997), o papel das instituições para o progresso técnico e a formação de inovações ficou evidenciado na teoria Schumpeteriana.

Apesar dos diferentes contextos e interpretações da palavra instituição na teoria econômica, a visão Schumpeteriana afirma que o progresso tecnológico depende da relação dos atores econômicos com o ambiente institucional voltado ao desenvolvimento das inovações, ou seja, da relação dos mesmos com o ambiente social. Como o intuito destes agentes é desenvolver o aprendizado $^{4}$, a interação com o ambiente pode ser sintetizada como um processo de captação de conhecimento (externo) para a geração de inovações.

Cimoli et alli (2003, p. 12) mostram que o processo de industrialização demanda uma maior presença de alguns atores institucionais, com destaque para o sistema financeiro, as universidades e o governo. A função destes atores está ligada ao desenvolvimento de novas tecnologias, para os autores:

(...) in the field of technology, public R\&D labs and State universities, together with public development banks that furnished long-term finance for major undertakings in fields such as energy, telecommunications and transport services, have played an important role the industrialization process. Public firms engaged in these kinds of activities developed their own engineering departments and R\&D facilities in order to supply themselves with the technology they needed for these ventures. As a complementary measure, public policies designed to attract foreign direct investment to manufacturing activities helped to obtain the required know-how in many technology-intensive sectors of the economy, such as the pharmaceuticals and auto3 Considera-se nessa afirmativa os autores Clássicos (Smith, Ricardo) e Neoclássicos (Jevons, Walras, entre outros), bem como a Escola Austríaca (Hayek e Mises).

4 Busca e seleção nas palavras de Nelson e Winter (1982). Todavia, o processo de aprendizado é pertinente à presença de competências na empresa e sua relação com o ambiente, capital social e instituições. Esta relação não será desenvolvida neste artigo, apenas apresenta-se que a estratégia microeconômica pertence ao contexto e também depende do ambiente. Ver a literatura Schumpeteriana voltada aos sistemas de inovações, como Edquist e Johnson (1997). 
motive industries.

A necessidade de atores institucionais no desenvolvimento tecnológico de uma economia industrializada reside no fato, segundo Nelson e Nelson (2002), de o processo de inovação depender do desenvolvimento tecnológico que pode ser físico ou social. A tecnologia física é tradicionalmente analisada pela teoria econômica, principalmente via capital físico que incorpora novos conhecimentos. A determinação da relação da tecnologia social com o desenvolvimento tecnológico pode ser considerada como uma grande contribuição Schumpeteriana. Ela é composta pela capacidade organizacional e pela cooperação da infraestrutura disponível ou necessidade de construção da mesma.

A geração de conhecimento tecnológico depende da utilização dessa infraestrutura, seja física ou social, que não está sob a posse de uma única empresa, pois sua utilização depende da construção de uma rede de atores, como universidades, governo, centros de pesquisa, ou mesmo de outras empresas (concorrentes, distribuidores, fornecedores, fabricantes de bens de capital, equipamentos ou insumos).

A geração de tecnologias sociais reforça a ideia da necessidade dos capitais social e humano no processo de inovação. Pode-se afirmar que o papel das instituições é de reforçar a interação entre o capital humano da empresa e o capital social do ambiente. A tríade - instituições, capital humano e capital social - é responsável pela formação de novos conhecimentos que impulsionarão o progresso técnico e a geração de inovações.

A relação entre as instituições e os dois tipos de capital gera os efeitos de transbordamento ${ }^{5}$ verificados na literatura, como em Nelson e Phelps (1966) e Dosi (1982). Estes transbordamentos refletem a incorporação e a valorização do conhecimento na atividade econômica, por meio dos dois tipos de capital e das instituições.

A necessidade de instituições no ambiente não reside apenas no tamanho e na escala ${ }^{6}$, mas principalmente pela complexidade exigida para o desenvolvimento das inovações, o que demanda domínio de conhecimentos tecnológicos, muitas vezes longe das competências verificadas nas empresas. Como Dosi, Orsenigo e Silberberg (1988) mostram que o conhecimento tecnológico pode ser caracterizado pelas seguintes qualidades: i) apropriabilidade, ii) incerteza, iii) oportunidade, iv) oportunidade comercial, v) cumulativo, vi) tácito; percebe-se que o papel das instituições é direcionar o comportamento dos agentes econômicos (empresas, fornecedores) e sociais (governo, universidades, pesquisadores) em relação as incertezas do processo de inovação.

A conclusão inerente a estas características do conhecimento tecnológico é a complexidade que permeia o seu desenvolvimento, o que conduz os indivíduos 
a apresentarem racionalidade limitada frente às decisões relativas à escolha do nível de investimento em determinada atividade inovadora. Por sua vez, o papel das instituições se revela como uma estratégia de aquisição de conhecimento por parte das empresas que não podem acumular conhecimento e competências suficientes para decidir de maneira precisa suas dúvidas sobre este investimento. As características apresentadas anteriormente mostram justamente o caráter sistêmico das inovações e de sua natureza social que varia desde a formação das ideias, até o desenvolvimento das mesmas sobre a forma de conhecimento e sua aplicação no campo econômico sob a forma de inovações, bem como a aceitação de consumidores, governantes e outros atores sociais dos novos produtos e serviços ofertados.

Neste sentido, o conhecimento que a empresa busca pode apresentar diferentes níveis de apropriação. O conhecimento pode pertencer a outro indivíduo ou empresa, ou ele depende de um elemento externo, como uma universidade, para decodificá-lo e transformá-lo em capacidade tácita aplicada a uma determinada tecnologia. A empresa, de acordo com o conhecimento e capacidades acumulados, estabelece uma relação com o ambiente por meio das instituições, com o intuito de desenvolver sua capacidade de absorção de conhecimento.

Este processo é complexo na medida em que sua previsão não é certa, apesar da trajetória seguida por uma determinada tecnologia, o estado da arte da mesma depende do acúmulo de conhecimento até o momento e das oportunidades, estratégica e comercial, sentidas pelos agentes econômicos. Este alto grau de incerteza é aprofundado pela competição entre diversas tecnologias e seus resultados que não seguem um padrão previsível7.

As características do conhecimento tecnológico geram falhas de mercado, em virtude da incerteza e dos resultados específicos para cada indivíduo que não são facilmente generalizados. Dosi (1988) mostra que o progresso tecnológico e a introdução de inovações estão cercados por falhas de mercado, compostas por externalidades, racionalidade limitada dos agentes e mercados imperfeitos.

Na literatura Schumpeteriana estas falhas de mercado são formadas pelo processo de mudança tecnológica inerente ao mercado e são a regra principal do funcionamento do mercado e não uma exceção. Neste sentido, elas são responsáveis, conforme Antonelli e Teubal (2008), pela formação de novas instituições voltadas ao progresso tecnológico, denominadas pelos autores como venture capitalism ${ }^{8}$. Em outras palavras, a estrutura econômica e social formada para gerar o progresso tecnológico é adaptada a uma realidade de

\footnotetext{
7 Torna-se importante destacar que a ideia de trajetória tecnológica não reduz a incerteza da tecnologia, na media em que à mesma não segue a ideia de previsão futura da chegada de novos conhecimentos. Ela diz que o estoque de conhecimento passado contribui para a formação de conhecimento novo, desta forma, o conhecimento é interligado e pode ser determinado endogenamente, portanto, ela não 'nasce' sem uma base passada de conhecimento acumulado pertencente a um paradigma tecnológico que engloba diversas trajetórias tecnológicas em diferentes estágios de desenvolvimento. Ver a discussão de Geroski (2000) em relação aos modelos de disseminação das inovações.

8 A tradução literal seria de capitalismo de risco.
} 
STEINGRABER, R. Empresa e Instituições na Teoria Schumpeteriana: Influências do Ambiente....

riscos e incerteza que são formadas pela necessidade de se vender informações (conhecimento).

A posição defendida por Antonelli e Teubal (2008) afirma que as instituições são dinâmicas, ou seja, elas evoluem conforme a demanda de conhecimento e a estrutura produtiva necessária para competir no mercado. Esta verificação é pertinente com a visão dos historiadores da tecnologia, como Rosenberg (1982) e Mowery e Rosenberg (2005). Para o autor, o progresso tecnológico depende de uma estrutura institucional cada vez mais complexa e dedicada ao progresso tecnológico e ao lançamento de inovações. King e Levine (1993) também mostram que as instituições, no caso as instituições voltadas para o financiamento da estrutura tecnológica e produtiva, evoluem e são formadas conforme as necessidades e incertezas do mercado.

Neste sentido, as instituições evoluem no ambiente conforme a estrutura geradora de novos conhecimentos e duas observações são identificadas. A primeira é a adaptação das instituições ao perfil da tecnologia, do setor econômico, indústria, e dos agentes externos envolvidos com a geração e difusão do conhecimento. A segunda é a ideia que as instituições também evoluem assim como os agentes impactados com a introdução de inovações, ou seja, a "destruição criadora" não afeta somente o capital físico e as empresas, mas igualmente o estoque de conhecimento disponível no ambiente. Como o conhecimento é cumulativo, a sua formação também é substituída (destruída), bem como as instituições envolvidas no processo. Este conceito vai ao encontro ao conceito de tecnologia social de Nelson e Sampat (2001) ao afirmar que o progresso técnico depende em grande medida de atores sociais, assim como fatores sociais não disponíveis no mercado (como as redes informais de interação entre os indivíduos).

O conceito de capital social, desenvolvido na próxima subseção, ajuda na definição do papel e alcance das instituições no processo de inovação, evitando-se a utilização do termo instituição para definir todos os atores, normas e convenções de caráter social.

\section{Inovação e capital social}

A utilização do termo capital social é nova na economia e reflete uma tentativa de aproximação da sociologia 9 . O objetivo da utilização do conceito de capital social visa justamente sobrepor o intenso e muitas vezes errático uso do termo instituição na teoria econômica, conforme defende Nelson e Sampat (2001).

Para existir uma 'produção social de conhecimento', deve-se formar de antemão os 'fatores' responsáveis por tal produção. Estes fatores são denominados de instituições e podem possuir estrutura física e conhecimento incorporado, eles também são regras e valores sociais que influenciam o comportamento 9 Ciência Política que cunhou o termo pela primeira vez. 
STEINGRABER, R. Empresa e Instituições na Teoria Schumpeteriana: Influências do Ambiente....

dos agentes econômicos. A ideia de capital social está associada ao relacionamento que pode ser estabelecido entre as instituições e os atores econômicos e sociais, gerando um aumento do volume de conhecimento e principalmente a sua difusão, ao formar uma 'rede' de atores.

Torna-se necessário restringir o termo capital social. Assim como o termo instituições, a definição de capital social é ampla e pode assumir significados diferentes, além ser usada em diferentes contextos. Putnam (2001) compara a controvérsia do capital de Cambridge com a utilização do termo capital social atualmente na literatura econômica. $\mathrm{O}$ autor defende que a definição e a extensão do termo capital social sofrem da mesma indeterminação do capital físico presente nas discussões da definição metodológica do conceito de capital da controvérsia de Cambridge ${ }^{10}$.

Putman (2001) ainda argumenta que a definição de capital social não deve ser confundida com filantropia ou altruísmo. Ela envolve a potencialização dos processos de crescimento e de desenvolvimento econômico, em função das informações advindas da interação entre os indivíduos na sociedade. Esta interação pode ser formal ou informal. No primeiro caso, apresenta-se o capital social formal, como a participação em um sindicato. O segundo caso, denominado de capital social informal, envolve a participação do indivíduo em situações esporádicas ou sem necessidade de formalização ou de repetição.

Em relação a esta discussão conceitual, Coleman (1988, p. s98) define capital social como:

It is not a single entity but a variety of different entities, with two elements in common: that all consist of some aspect of social structures, and they facilitate certain actions of actors - whether persons or corporate actors - within the structure. Like other forms of capital, social capital is productive, making possible the achievement of certain ends that in its absence would not be possible. Like physical capital and human capital, social capital is not completely fungible but may be specific to certain activities. A given form of social capital that is valuable in facilitating certain actions may be useless or even harmful for others.

A partir desta definição, percebe-se que a conexão entre os indivíduos é vital para o sucesso econômico da inovação. A estrutura social presente no ambiente

10 Esta discussão conhecida como "controvérsia do capital", conforme Cohen e Harcourt (2003), envolveu as duas escolas de economia de Cambridge: a inglesa, com Piero Sraffa, Jon Robinson, Luigi Pasinetti e Pierangelo Garegnani e a norte-americana, com Paul Samuelson, Robert Solow, Frank Hahn e Chritopher Bliss. A discussão envolveu a definição do conceito de capital na função de produção agregada. Os pesquisadores de Cambridge no Reino Unido discordavam da forma como seus pares nos Estados Unidos definiram a mensuração agregada do capital, que envolve o valor monetário do capital empregado, todavia, em atividades econômicas diferentes o capital possui utilidades diferentes e assume formas e níveis tecnológicos que são desconsiderados na função agregada. 
STEINGRABER, R. Empresa e Instituições na Teoria Schumpeteriana: Influências do Ambiente....

onde os indivíduos partilharão de regras e valores, bem como a reputação dos mesmos forma o capital social.

As relações que formam o capital social são classificadas basicamente em duas formas. A primeira é da densidade. A segunda é da extensão.

A densidade explica o grau de envolvimento dos indivíduos com a rede. Ela avalia o compromisso dos indivíduos com o ambiente social e os demais indivíduos. Se o capital social for denso, como uma relação familiar, por exemplo, o indivíduo possui um forte alinhamento de seu comportamento e decisões com os valores, regras e reputação do grupo.

Se o capital social for pouco denso, o comprometimento dos indivíduos com as informações do ambiente serão de pouca, ou pequena, relevância. O impacto econômico do capital social será maior em função da densidade do mesmo. Sob este ponto de vista, quanto maior a densidade da relação entre os agentes econômicos (empresas) e as instituições (do ambiente social, como uma universidade), maior será a probabilidade de sucesso econômico, em função do aumento do fluxo de informações entre as partes.

Analisando-se a formação e a transferência de conhecimento que alimenta a formação de inovações na economia, quanto mais densa a relação entre as empresas e as instituições, maior a geração de conhecimento. A difusão tecnológica será igualmente maior, o que aumenta as chances de imitação da inovação, reduzindo o ciclo de vida do produto inovador e aumentando a sua apropriação, por parte de terceiros.

As características do conhecimento analisadas em Dosi et alli (1988) podem ser consideradas como a formação de capital social a partir das características da tecnologia. Quanto mais complexo e tácito for o conhecimento da tecnologia mais denso será o capital social exigido. O mesmo raciocínio pode ser desenvolvido para as características de oportunidades relacionadas ao conhecimento de determinada tecnologia, estas oportunidades são dadas pelas preferências dos consumidores, definidas por características socioeconômicas.

Em relação à extensão do capital social, ela mostra o número de indivíduos que pertencem à determinada rede. $\mathrm{O}$ impacto econômico torna-se maior proporcionalmente ao tamanho da rede, mas não existe regra no sentido de que redes maiores sempre serão mais benéficas economicamente. A extensão do capital social ajuda no desenvolvimento tecnológico primeiramente porque o conhecimento tecnológico é cumulativo e um número maior de indivíduos ajudará na geração de novas ideias e aplicações do conhecimento. Em segundo lugar, quanto maior a rede de agentes que adotam um novo conhecimento, maior o desenvolvimento da tecnologia em função do aumento da escala ${ }^{11}$. Alguns estudos, como Dosi (1988), mostram que a rede de adoção de um novo

11 Os benefícios da escala (número de indivíduos que adotam uma determinada tecnologia) podem apresentar uma distribuição quadrática, com benefícios que se esgotam a partir de determinado tamanho, conduzindo a retornos decrescentes de escala. 
STEINGRABER, R. Empresa e Instituições na Teoria Schumpeteriana: Influências do Ambiente....

conhecimento é decisiva para o sucesso de uma inovação, na medida em que a mesma está inserida em um ambiente de competição entre diversos novos conhecimentos.

Dasgupta e Serageldin (2000) analisam o conceito de capital social e, para os autores, o termo capital significa algo tangível e a utilização do mesmo para definir relações sociais intangíveis pode causar confusão. Apesar da discussão relacionada à utilização do termo capital para fatores sociais, a aplicação do conceito de capital social abriu importantes perspectivas na Economia, pois as relações entre os atores sociais, as empresas e as instituições eram denominadas de instituições e causavam confusão, ou seja, a empresa para inovar necessita interagir com as instituições (atores sociais), conforme as instituições vigentes (regras sociais, formais e informais), a rotina dessas relações estabeleciam novas instituições para inovar (na verdade, eles formavam capital social). Apesar da contribuição do conceito de capital social sobre a definição de instituição na teoria Schumpeteriana, ainda se percebe a sobreposição do termo instituição em relação aos atores sociais envolvidos no processo de inovação.

Para que o conceito de capital social não esteja sobrepondo o conceito de instituição, deve-se considerar a definição de capital social de Knack e Keefer (1997, p. 1251). Para os autores o capital social compreende:

i) The relationship between interpersonal trust, norms of civic cooperation, and economic performance, and some of the policy and other links through which these dimensions of social capital may have economic effects;

ii) The conflicting hypotheses of Putnam (1993) and Olson (1982), on the relationship between associational activity and growth; and

iii) The determinants of trust and norms of civic cooperation, including levels of associational activity and formal institutions.

A definição apresentada por Knack e Keefer (1997) reforça a ideia de que o capital social é composto por fatores não econômicos que podem ser coletivos (sociais), como o conflito no associativismo, ou individuais, como a confiança pessoal.

Estas definições vão ao encontro do conceito de venture capitalism de Antonelli e Teubal (2008). Os autores explicam que a economia da inovação e do progresso tecnológico demanda novas instituições para o seu desenvolvimento, no sentido de um arcabouço institucional e de novas regras de comportamento e de relacionamentos. Em outras palavras, para que a inovação ganhe corpo no mercado, o capital social deve ser estabelecido, conectando as empresas com as instituições.

As falhas de mercado apontadas por Dosi (1988) também podem ser explica- 
das pela dificuldade de formação de capital social no processo de inovação, principalmente em função da aplicação do conceito de racionalidade limitada ${ }^{12}$ que surge em função das limitações dos mecanismos de busca de informações, conforme Nelson e Winter (1982), o que torna o aprendizado do indivíduo dependente da interação com o ambiente. As empresas são igualmente analisadas como organizações, compostas por diversos interesses e atores. Em relação à organização, Nelson e Nelson (2002) discutem a necessidade de inovação organizacional frente ao progresso tecnológico, o que mostra a existência de aprendizado coletivo, organizacional, originado na formação de capital social para a viabilidade do processo de inovação nas empresas, além de que o capital social estabelecido é diferente em função das características próprias dos indivíduos e das instituições, em outras palavras, o capital social é diferente em função da forma particular como os indivíduos se relacionam com as instituições para inovar.

Outra aplicação do capital social é a relação universidade-empresa. A universidade é citada como uma instituição de geração de conhecimento e que é responsável pelo aumento das chances de inovação na economia. Por esta razão, Nelson e Rosenberg (1993, p. 15) destacam o papel privilegiado da universidade como fator de progresso tecnológico, para os autores "importantes interações e redes, não são as mesmas em todas as indústrias ou tecnologias".

O papel de destaque da universidade como instituição de promoção do progresso tecnológico está no capital social apresentado na relação entre as empresas e a universidade. Esta relação pode ser de densidade ou de extensão. No primeiro caso, a relação reside no tipo de conhecimento necessário para o desenvolvimento de inovações, na visão dos autores, cada vez mais as empresas dependem das universidades para inovarem, o que significa que a relação universidade-empresa necessita de adensamento para o sucesso da inovação e da difusão de novos conhecimentos. A relação de extensão mostra que o aumento do número de empresas reforça a trajetória de uma determinada tecnologia, reduzindo os riscos de adoção. A extensão determina que quanto mais distante a empresa se situe da universidade, mais fraca será a relação, o que segundo Audretsch et alli (2005, p. 1120-1121) explicaria a presença do efeito de transbordamento do conhecimento, restrito geograficamente ao redor da universidade. A restrição geográfica do efeito de transbordamento do conhecimento da universidade e o aumento da probabilidade de inovação das empresas é função do capital social estabelecido entre a universidade e as empresas onde:

(...) new firms in high-technology industries are influenced not only by the traditional regional characteristics, but also by the opportunity to access knowledge generated by universities. gias, produtos e processos necessários para a decisão de inovar. A racionalidade pode ser limitada também em função de dificuldades organizacionais que surgem no processo de inovação. 
(...) the impact of university output on new firm location is sensitive to both the type of knowledge and mechanism used to access that knowledge. Thus, the role of geographic proximity to access university knowledge in not simple and straightforward, but rather depends on the knowledge type and the spillover mechanism.

Não somente o tipo de conhecimento é relevante, conforme apontam Dosi et alli (1988), mas também o forma como este conhecimento estabelece a relação entre a universidade e as empresas. A formação de capital social entre a universidade e as empresas é limitada pela localização e pela própria característica da tecnologia envolvida. Tecnologias relativamente novas (na fase inicial de sua trajetória) abrem novas oportunidades, o que facilita o que Audretsch et alli (2005) denominam de empreendedorismo tecnológico, relacionado com o desenvolvimento de novos conhecimentos e suportado por meio de políticas públicas e oportunidades percebidas pelos empresários empreendedores.

O papel do capital social na determinação do papel da universidade como uma instituição do apoio à inovação é evidente. Primeiramente, por meio da geração de capital humano para a atividade de inovação. Coleman (1988) e Knack e Keefer (1997) reforçam a ideia de que o capital social é um importante fator na construção de capital humano para a sociedade. Conforme Nelson e Rosenberg (1993) a conexão entre o capital humano presente nas empresas e nas universidades pode ser denominada de capital social e tende a ser o principal fator de referência para a geração de novos conhecimentos.

Estes novos conhecimentos geram a oportunidade de novos negócios. As novas oportunidades tecnológicas são exploradas pelo empreendedor e refletem a estrutura institucional limitada espacialmente e a rede de difusão de conhecimento entre a universidade e as empresas e novos empreendedores (Audretsch, 1998; Audretsch e Feldman, 1996).

A simples presença de universidades em um determinado local não gera aumento do número de inovações, tão pouco aumenta o valor econômico das mesmas. A relação estabelecida entre a instituição universidade e a geração de capital humano para as empresas é que abre novas oportunidades de negócio e determina o aumento da qualidade das inovações, portanto, o valor da inovação reflete o valor do capital social estabelecido no processo de inovação.

O capital humano, gerado pelas universidades, permeia as empresas e aumenta a conexão entre as mesmas. As empresas também geram capital humano, seja pelo treinamento disponibilizado, seja pelo conhecimento tácito, evidenciado pelo aprendizado de fazer (learning by doing). A complementariedade entre o conhecimento codificado e o tácito permeia a importância do capital social, na medida em que aproxima os atores responsáveis pelas diversas fases do processo de inovação. 


\section{Considerações finais}

Este artigo identificou a apropriação do conceito de capital social e a evolução do conceito de instituição na teoria Schumpeteriana. Percebe-se que a tecnologia é vista socialmente nesta escola de pensamento econômico, o que a aproxima de outros campos do conhecimento, como a Sociologia e a História.

Primeiramente, o termo instituição representou esta aproximação, ao definir que atores externos e as regras do jogo, formais e informais, são responsáveis pela formação do conhecimento tecnológico, que é a matéria prima para a formação de inovações, portanto, a empresa inovadora precisa interagir com o ambiente econômico e social para avançar no processo de inovação.

Justamente nesse processo de interação que surge a aplicação do termo capital social. Esta ligação também é responsável pelo sucesso do processo de inovação na Economia e varia em densidade e extensão, além da informalidade, que assim como as instituições, é uma característica social a ser considerada, pois nem todas as regras sociais estão escritas (formalizadas).

Percebe-se que a escola Schumpeteriana ainda está avançando em relação à construção e aplicação dos conceitos de instituições e capital social no processo de inovação, mas não se discute a importância dos mesmos neste processo, visto que vários estudos apresentados destacam a necessidade desta relação, bem como o seu caráter social.

De forma sintética, percebe-se que o processo de inovação não pode ser reduzido a uma análise objetiva e racional do mercado, agregando elementos do ambiente social envolvidos naturalmente na formação de conhecimento e na sua aplicação e uso no mercado. Neste sentido, a visão apresentada de inovação é mais holística em relação às escolas ortodoxas de Economia e envolve mais atores, inclui-se aqui os sociais, e os resultados alcançados não podem ser considerados ótimos, pelo contrário, a participação das instituições e do capital social denota que o processo de inovação é dinâmico e complexo, ao contrário da visão determinística de equilíbrio das escolas ortodoxas clássica e neoclássica. Percebe-se que a escola Schumpeteriana evoluiu ao introduzir as instituições e o capital social na sua análise do processo de inovação, todavia, estudos futuros devem estabelecer um diálogo mais intenso entre as definições desta escola e a Sociologia e áreas afins.

\section{Referências Bibliográficas}

Antonelli, C; Teubal, M (2008). Knowledge-intensive property rights and the evolution of venture capitalism. Journal of Institutional Economics, volume 4, number 2, p. 163-182.

Audretsch, D. B; Feldman, M. P (1996). Innovative clusters and the industry life 
STEINGRABER, R. Empresa e Instituições na Teoria Schumpeteriana: Influências do Ambiente....

cycle. Review of Industrial Organization, volume 11, p. 253-273.

Audretschi, D. B (1998). Agglomeration and the location of innovative activity. Oxford Review of Economic Policy, volume 14, number 2.

Audretsch, D. B; Lehmann, E. E (2005). Does the knowledge spillover theory of entrepreneurship hold for regions? Research Policy, volume 34, issue 8, p. 1191-1202.

Cimoli, M; Correa, N; Katz, J; Studart, R (2003). Institutional requirements for market-led development in Latin America. Santiago: CEPAL - series informes y estudios especiales.

Cohen, A. J; Harcourt, G. C (2003). Whatever happened to the Cambridge capital theory controversies? Journal of Economic Perspectives, volume 17, number 1, p. 199-214.

Coleman, J. S (1998). Social capital in the creation of human capital. The American Journal of Sociology, volume 94 - Supplement: Organizations and Institutions: sociological and economic approaches to the analysis of social structure, p. S95-S120.

Dasgupta, P; Serageldin, I (200o). Social capital: a multifaceted perspective. Washington: World Bank.

Dosi, G (1982). Technological paradigms and technological trajectories: a suggested interpretation of the determinants and direction of the technical change. $R e$ search Policy, volume 11, p. 147-162.

Dosi, G (1988). Institutions and markets in a dynamic world. The Manchester School of Economics \& Social Studies, volume 56, number 2, p. 119-146.

Dosi, G; Orsenigo, L; Silverberg, G (1988). Innovation, diversity and diffusion: a self-organization model. The Economic Journal, 98, p. 1032-1054.

Edquist, C (1997). Systems of innovation: technologies, institutions, and organizations. London: Routledge.

Edquist, C; Johnson, B (1997). Institutions and organizations in systems of innovation. In: EDQUIST, C. Systems of innovation: technologies, institutions, and organizations. London: Routledge, p. 41-63.

Geroski, P. A (2000). Models of technology diffusion. Research Policy, volume 29, p.603-625.

King, R. G; Levine, R (1993). Finance and growth: Schumpeter might be right. Quarterly Journal of Economics, volume 108, issue 3, p. 717-737.

Knack, S; Keefer, P (1997). Does social capital have an economic payoff? A crosscountry investigators. The Quarterly Journal of Economics, volume 112, number 4, p. 1251-1288.

Mowery, D. C; Rosenberg, N (2005). Trajetórias da inovação: a mudança tecnológica nos Estados Unidos da América no século XX. Campinas: Unicamp.

Nelson, R. R; Phelps, E. S (1966). Investments in humans, technological diffusion, and economic growth. The American Economic Review, volume 56, number $1 / 2$, p. 69-75.

Nelson, R. R; Rosemberg, N (1993). Technical innovation and national systems. In: NELSON, RR. National innovation systems: a comparative analysis. Oxford: 
STEINGRABER, R. Empresa e Instituições na Teoria Schumpeteriana: Influências do Ambiente...

Oxford University, p. 3-21.

Nelson, R. R; Sampat, B. N (2001). Making sense of institutions as a factor shaping economic performance. Journal of Economic Behavior \& Organization, volume 44, p. 31-54.

Nelson, R. R; Nelson, K (2002). Technology, institutions, and innovation system. Research Policy, volume 31, p.265-272.

Nelson, R. R; Winter, S (1982). An evolutionary theory of economic change. Cambridge: Cambridge University Press.

Putnam, R (2001). Social capital: measurement and consequences. Paris: OECD.

Rosenberg, N (1982). Inside the black box: technology and economics. New York: Cambridge University.

Olson, M (1982). The rise and decline of nations: economic growth, stagnaflation, and social rigidities. Yale: Yale University Press.

Recebido em: 13 de dezembro de 2012 Aceito em: 11 de março de 2013 
STEINGRABER, R. Empresa e Instituições na Teoria Schumpeteriana: Influências do Ambiente.... 